\title{
Design, Synthesis and Pharmacological Evaluation of New Coumarin Derivatives as Monoamine Oxidase A and B Inhibitors
}

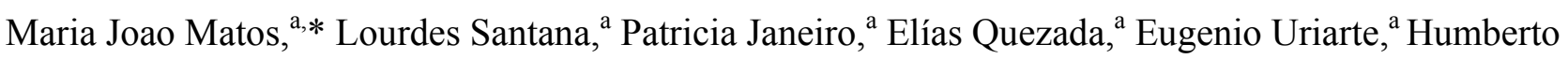 \\ González-Díaz, ${ }^{\mathrm{b}}$ Dolores Viña, ${ }^{\mathrm{c}}$ and Francisco Orallo ${ }^{\mathrm{c}}$ \\ ${ }^{a}$ Department of Organic Chemistry, ${ }^{b}$ Department of Parasitology and ${ }^{c}$ Department of Pharmacology, \\ Faculty of Pharmacy, University of Santiago de Compostela 15782, Spain.
}

\begin{abstract}
“Abstract." With the aim to find out the structural features for the MAO inhibitory activity and selectivity, in the present communication we report the design, synthesis and pharmacological evaluation of a new series of coumarin derivatives with 4-methyl or cycloalkene or benzene ring condensed in the 3,4 position. The substituents in this new scaffold were introduced in the 5, 7 and/or 8 positions of the coumarin moiety. The synthesized compounds 1-13 were evaluated as MAO A and B inhibitors using clorgyline and selegiline, respectively, as reference inhibitors, showing, most of them, activities in the nanomolar range. Compounds $\mathbf{6}\left(\mathrm{IC}_{50}=1.18 \mathrm{nM}\right)$ and $\mathbf{1 0}\left(\mathrm{IC}_{50}=1.48 \mathrm{nM}\right)$, show higher activity than selegiline $\left(\mathrm{IC}_{50}=19.60 \mathrm{nM}\right)$, and high MAO-B selectivity with 100 -fold and 1600 -fold inhibition levels, with respect to the MAO-A isoform.
\end{abstract}

*To whom correspondence should be addressed - e-mail: mariajoao.correiapinto@,rai.usc.es

\section{Introduction}

Mono amine oxidases (MAOs) are flavoenzymes bound to the outer mitochondrial membrane and are responsible for the oxidative deamination of neurotransmitters and dietary amines. ${ }^{1,2}$ Two isoforms, namely MAO-A and MAO-B, have been identified on the basis of their amino acid sequences, threedimensional structure, substrate preference and inhibitor selectivity. ${ }^{3,4} \mathrm{MAO}-\mathrm{A}$ has a higher affinity for 
serotonin and noradrenaline whereas MAO-B preferentially deaminates phenylethylamine and benzylamine. These properties determine the clinical importance of MAO inhibitors. Selective MAO-A inhibitors such as clorgyline (irreversible) and moclobemide (reversible) are used in the treatment of neurological disorders such as depression, ${ }^{5,6}$ whereas the selective and irreversible MAO-B inhibitors such as selegiline and rasagiline are useful in the treatment of Parkinson's ${ }^{7,8}$ and Alzheimer's diseases. $^{9,10}$

All of these aspects have led to an intensive search for novel MAO inhibitors (MAOIs) and this effort has increased considerably in recent years. However, earlier MAOIs introduced into clinical practice were abandoned due to adverse effects, such as hepatotoxicity, orthostatic hypotension and the so-called “cheese effect", which was characterized by hypertensive crisis. ${ }^{11,12}$

In recent years, a broad consensus has been reached concerning the necessity for a search for novel MAOIs and the study of their interaction with MAOs. ${ }^{13-15}$ The major breakthrough has been brought about by the crystallization of hMAO-B with different inhibitors. ${ }^{16}$ This fact explains the subsequent elucidation and determination of the $3 \mathrm{D}$ structure of the active site of hMAO- $\mathrm{A}^{3}$ and opened new possibilities for the design of more selective and reversible drugs and facilitated the computer-assisted development of more selective inhibitors. Molecular docking calculations, ${ }^{17,18}$ comparative molecular field analysis (CoMFA) $)^{19,20}$ as well as QSAR methods are among the computational methods that have been used to predict MAOIs. Respect of this, in previous works our group has developed a new QSAR methodology called MARCH-INSIDE ${ }^{21,22}$ methodology to seek theoretical models for the prediction of different biological activities. Applying this method is possible to predict the in vitro MAO A/B inhibitory activity of new series of coumarin derivatives.

A specific way to new lead discovery involves several general common steps when used in terms of a QSAR: (a) construction of a suitable molecular database of compounds that either show the property in question or do not; (b) calculation of the molecular descriptors; (c) construction of the model; (d) estimation of the biological activity using QSAR; (e) synthesis and characterization of selected 
compounds; (f) assay of the candidate compounds in order to corroborate the predicted biological activity.

The coumarin analogs are a family of natural and/or synthetic compounds with different pharmacological activities, one of which is MAO inhibitory activity. ${ }^{23,24}$ In many cases, it is known that activity and selectivity are determined by the nature of the substituents at the 7 - and the 4/3-positions. ${ }^{25-}$ ${ }^{27}$ On the other hand, in a previous investigation ${ }^{28}$ we developed QSAR studies for the MAO-A inhibitory activity in a series of coumarin derivatives and found that the most active compounds were the 7-acetonyloxy-substituted compounds, which showed higher activity than their cyclic analogs and were also more active than the 7-hydroxy precursors.

\section{Results and discussion}

The theoretical QSAR model was created using a very large data base of heterogeneous compounds in which activities were measured in cellular lines from different organs and species. The model classified correctly 3222 out of 3408 inputs $(94.5 \%)$ and was then used to predict the activity of 13 compounds in our designed coumarin library. All of the active compounds were correctly predicted by the model, and a total of $81.82 \%$ and $72.73 \%$ compounds were correctly predicted as MAO-A and MAO-B inhibitors, respectively.

On the basis of the QSAR model information - and with the aim of exploring the structure-affinity and MAO-A/B selectivity relationships - in the present work we designed a series of 13 coumarin derivatives (Table 1) that include the aforementioned chemical diversity with particular attention paid to the most interesting 7-( $\beta$-ketoether)coumarin derivatives. The compounds were synthesized according to Scheme 1 and details are given in the Experimental section.

The SAR analysis seems to corroborate the importance of the structural requirements of the substituents in the coumarin moiety in order to achieve good activity and MAO-A/B selectivity, as indicated in the literature. ${ }^{24-26}$ The 7-hydroxy derivatives have been shown to be inactive, even with the 
introduction of bulky substituents in any other positions of the coumarin (compounds $\mathbf{1}$ and 2). The introduction of acetonyl/bromoallyloxy groups in the 7-position of the coumarin yielded compounds (3 to 10) with greater MAO-A and MAO-B inhibitory activity. The substitution in the 5 position (compounds 11 and 12) of the coumarin ring leads to the activity loss either in the case of the ketoether substituent or the cyclation to the corresponding furocoumarin 13. Finally, the most relevant findings are the introduction of bulky groups such as cyclohexyl or phenyl in the 3,4-positions of the 7-acetonyl derivatives increased both MAO-A and MAO-B inhibitory activities with concomitant loss of selectivity, whereas replacement of the acetonyl substituent at position 7 by the bromoallyloxy group resulted in compounds $\mathbf{6}$ and 10, which had very high MAO-B inhibitory activity $\left(\mathrm{IC}_{50}\right.$ of about $1.2 \mathrm{nM}$ and $1.5 \mathrm{nM}$ ) and the highest MAO-B selectivity (approximately 100-fold and 1600-fold) with respect to the MAO-A isoform.

Table 1. Structure of the coumarin derivatives 1-13

\begin{tabular}{|c|c|c|c|c|}
\hline Compound & \begin{tabular}{l|l}
$\mathrm{R}_{3}$ & $\mathrm{R}_{4}$
\end{tabular} & $\mathrm{R}_{5}$ & $\mathrm{R}_{7} /\left(\mathrm{CH}_{2}\right) \mathrm{n}$ & $\mathrm{R}_{8}$ \\
\hline 1 & $-\left(\mathrm{CH}_{2}\right)_{3}-$ & $\mathrm{H}$ & $\mathrm{OH}$ & $\mathrm{Me}$ \\
\hline 2 & $-\left(\mathrm{CH}_{2}\right)_{4}-$ & $\mathrm{H}$ & $\mathrm{OH}$ & $\mathrm{Me}$ \\
\hline 3 & $-\left(\mathrm{CH}_{2}\right)_{3}-$ & $\mathrm{H}$ & $\mathrm{MeCOCH}_{2} \mathrm{O}-$ & $\mathrm{Me}$ \\
\hline 4 & $-\left(\mathrm{CH}_{2}\right)_{4}-$ & $\mathrm{H}$ & $\mathrm{MeCOCH}_{2} \mathrm{O}-$ & $\mathrm{Me}$ \\
\hline 5 & $-\left(\mathrm{CH}_{2}\right)_{4}-$ & $\mathrm{H}$ & $\mathrm{MeCOCH}_{2} \mathrm{O}-$ & $\mathrm{OMe}$ \\
\hline 6 & $-\left(\mathrm{CH}_{2}\right)_{3}-$ & $\mathrm{H}$ & $\mathrm{CH}_{2} \mathrm{C}(\mathrm{Br}) \mathrm{CH}_{2}-$ & $\mathrm{Me}$ \\
\hline 7 & $-\left(\mathrm{CH}_{2}\right)_{4}-$ & $\mathrm{H}$ & $\mathrm{CH}_{2} \mathrm{C}(\mathrm{Br}) \mathrm{CH}_{2}-$ & Me \\
\hline 8 & $\mathrm{Ph}$ & $\mathrm{H}$ & $\mathrm{MeCOCH}_{2} \mathrm{O}-$ & $\mathrm{Me}$ \\
\hline
\end{tabular}




\begin{tabular}{|c|c|c|c|c|c|}
\hline $\mathbf{9}$ & \multicolumn{2}{|c|}{$\mathrm{Ph}$} & $\mathrm{H}$ & $\mathrm{MeCOCH}_{2} \mathrm{O}-$ & $\mathrm{OMe}$ \\
\hline $\mathbf{1 0}$ & \multicolumn{2}{|c|}{$\mathrm{Ph}$} & $\mathrm{H}$ & $\mathrm{CH}_{2} \mathrm{C}\left(\mathrm{Br}^{2} \mathrm{CH}_{2}-\right.$ & $\mathrm{Me}$ \\
\hline $\mathbf{1 1}$ & $\mathrm{H}$ & $\mathrm{CH}_{3}$ & $\mathrm{OH}$ & $\mathrm{OMe}$ & $\mathrm{H}$ \\
\hline $\mathbf{1 2}$ & $\mathrm{H}$ & $\mathrm{CH}_{3}$ & $\mathrm{MeCOCH}_{2} \mathrm{O}-$ & $\mathrm{OMe}$ & $\mathrm{H}$ \\
\hline $\mathbf{1 3}$ & \multicolumn{2}{|c|}{-} & - & - & - \\
\hline
\end{tabular}

Chemistry. The coumarin derivatives 1-13 were efficiently synthesized according to the synthetic protocol outlined in Scheme 1.

Pechmann condensation of 2-alkyl resorcinol with the corresponding ketoester afforded the 3,4cyclopentene/cyclohexene-7-hydroxy coumarins 1, 2 and 11. The Williamson reaction of the 7hydroxycoumarins 1, 2 and $\mathbf{1 1}$ with 2-chloroketones or 2,3-dibromopropene gave the corresponding ethers 3, 4, 6, 7 and 12. Compounds 4, 5 and 7 were oxidized with DDQ to give the corresponding 3,4benzocoumarin derivatives 8-10. Finally, the compound $\mathbf{1 2}$ was treated in basic conditions to obtain the furocoumarin 13. 


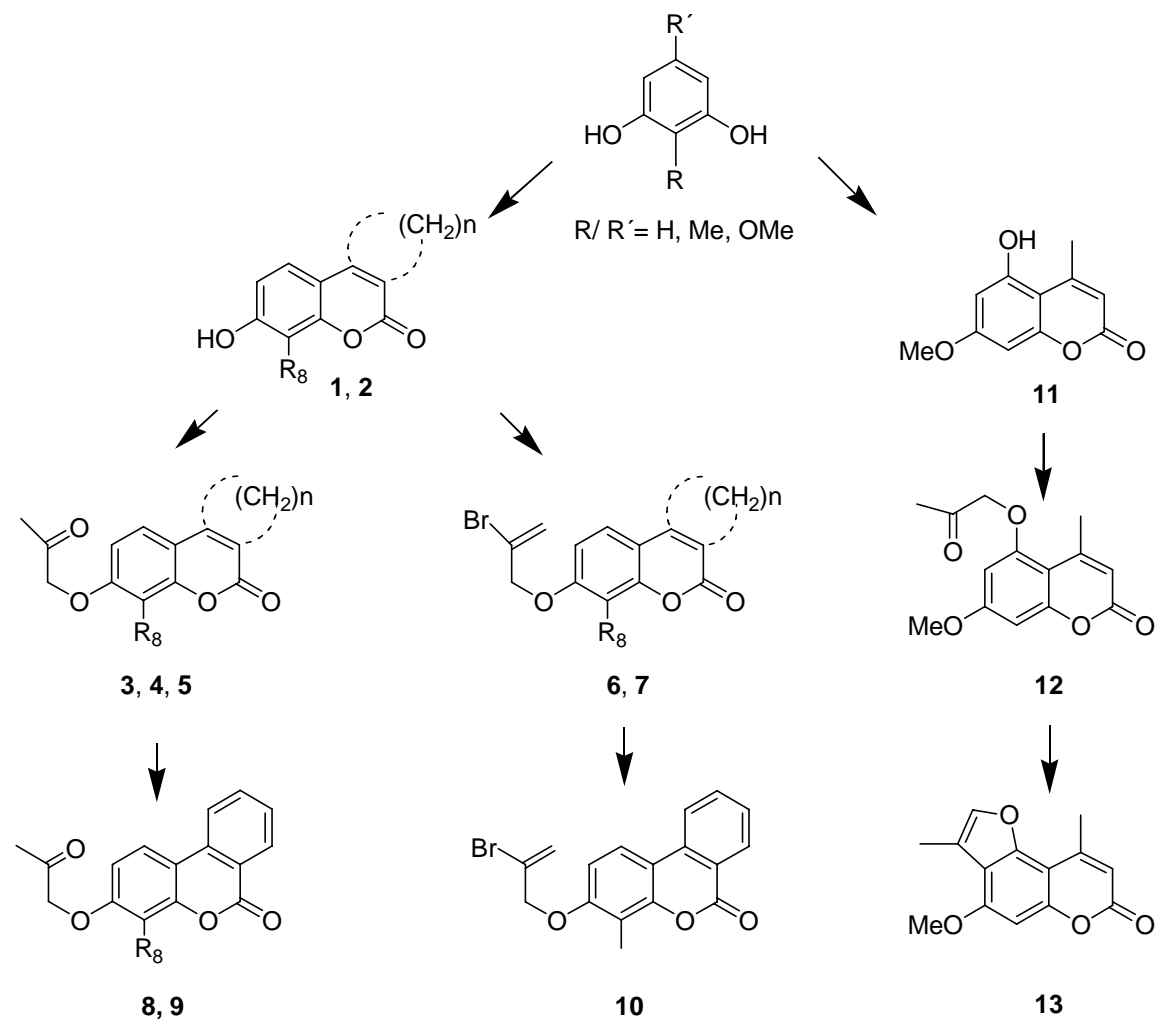

Scheme 1. Synthetic strategy for prepared compounds

MAO inhibition assay. The potential effects of the test drugs on hMAO activity were investigated by measuring their effects on the production of hydrogen peroxide from $p$-tyramine (a common substrate for both hMAO-A and hMAO-B), using the 10-acetyl-3,7-dihydroxyphenoxazine as reagent and microsomal MAO isoforms prepared from insect cells (BTI-TN-5B1-4) infected with recombinant baculovirus containing cDNA inserts for hMAO-A or hMAO-B.

The production of $\mathrm{H}_{2} \mathrm{O}_{2}$ catalyzed by $\mathrm{MAO}$ isoforms can be detected using the previously mentioned reagent, a non-fluorescent, highly sensitive and stable probe that reacts with $\mathrm{H}_{2} \mathrm{O}_{2}$ in the presence of horseradish peroxidase to produce a fluorescent product: resorufin. In this study hMAO activity was evaluated using the above method following the general procedure described previously by us. ${ }^{29}$

The tested drugs (new compounds and reference inhibitors) inhibited the control enzymatic MAO activities and the inhibition was concentration dependent. The corresponding $I C_{50}$ values and MAO-B 
selectivity ratios $\left[I C_{50}(\mathrm{MAO}-\mathrm{A})\right] /\left[I C_{50}(\mathrm{MAO}-\mathrm{B})\right]$ are shown in Table 2.

Table 2. In vitro evaluation of MAO inhibitory activities of compounds 1-13 and reference inhibitors

\begin{tabular}{|c|c|c|c|}
\hline & MAO-A & MAO-B & $\mathrm{MAO}-\mathrm{A} / \mathrm{B}$ \\
\hline Compound & $\mathrm{IC}_{50}$ & $\mathrm{IC}_{50}$ & \\
\hline 1 & $* *$ & $* *$ & - \\
\hline 2 & $60.11 \pm 3.47 \mu \mathrm{M}$ & $* *$ & $<0.60^{\mathrm{c}}$ \\
\hline 3 & $235 \pm 18.52 \mathrm{nM}^{\mathrm{b}}$ & $165 \pm 7.04 \mathrm{nM}$ & 1.42 \\
\hline 4 & $3.78 \pm 0.34 \mu \mathrm{M}^{\mathrm{a}}$ & $17.3 \pm 1.48 \mu \mathrm{M}$ & 0.22 \\
\hline 5 & $396 \pm 16.63 \mathrm{nM}^{\mathrm{a}}$ & $12.12 \pm 1.03 \mu \mathrm{M}$ & 0.033 \\
\hline 6 & $130 \pm 11.77 \mathrm{nM}^{\mathrm{a}}$ & $1.18 \pm 0.15 \mathrm{nM}$ & 110 \\
\hline 7 & $695 \pm 28.54 \mathrm{nM}^{\mathrm{b}}$ & $236 \pm 5.03 \mathrm{nM}$ & 2.94 \\
\hline 8 & $2.56 \pm 0.14 \mu \mathrm{M}^{\mathrm{a}}$ & $28.13 \pm 1.14 \mu \mathrm{M}$ & 0.091 \\
\hline 9 & $11.93 \pm 0.61 \mu \mathrm{M}$ & $9.36 \pm 0.26 \mu \mathrm{M}$ & 1.27 \\
\hline 10 & $2.38 \pm 0.06 \mu \mathrm{M}^{\mathrm{a}}$ & $1.49 \pm 0.12 \mathrm{nM}$ & 1,597 \\
\hline 11 & $>100 \mu \mathrm{M}$ & $>100 \mu \mathrm{M}$ & - \\
\hline 12 & $65,12 \mu \mathrm{M}$ & $>100 \mu \mathrm{M}$ & - \\
\hline 13 & $*$ & * & - \\
\hline Clorgyline & $4.46 \pm 0.32 \mathrm{nM}^{\mathrm{a}}$ & $61.35 \pm 1.13 \mu \mathrm{M}$ & 0.00007 \\
\hline Selegiline & $67.25 \pm 1.02 \mu \mathrm{M}^{\mathrm{a}}$ & $19.60 \pm 0.86 \mathrm{nM}$ & 3,431 \\
\hline Iproniazide & $6.56 \pm 0.76 \mu \mathrm{M}$ & $7.54 \pm 0.36 \mu \mathrm{M}$ & 0.87 \\
\hline Moclobemide & $361 \pm 19.37 \mu \mathrm{M}$ & $*$ & $<0.36^{\mathrm{c}}$ \\
\hline
\end{tabular}


Each $\mathrm{IC}_{50}$ value is the mean \pm S.E.M. from five experiments $(\mathrm{n}=5)$.

* Inactive at $1 \mathrm{mM}$ (highest concentration tested).

** Inactive at $100 \mu \mathrm{M}$ (highest concentration tested). At higher concentrations the compounds precipitate.

*** Inactive at $50 \mu \mathrm{M}$ (highest concentration tested). At higher concentrations the compounds precipitate.

${ }^{\mathrm{a}, \mathrm{b}}$ Level of statistical significance: ${ }^{\mathrm{b}} \mathrm{P}<0.01$ or ${ }^{\mathrm{b}} \mathrm{P}<0.05$ versus the corresponding $\mathrm{IC}_{50}$ values obtained against MAO-B as determined by ANOVA/Dunnett's.

${ }^{\mathrm{c}}$ Values obtained under the assumption that the corresponding $\mathrm{IC}_{50}$ against $\mathrm{MAO}-\mathrm{A} / \mathrm{B}$ is the highest concentration tested.

\section{Conclusions}

In summary, we have developed good theoretical models to predict inhibitory MAO activity. On the basis of this model we have found new coumarin derivatives with inhibitory activity comparable to those of clorgyline and selegiline, respectively, which are used as reference inhibitors and have a very high MAO-A and MAO-B selectivity. These findings have encouraged us to continue our investigations into the design of more potent and selective analogs by introducing appropriate substituents into the coumarin scaffold.

\section{Experimental section}

Chemistry. Melting points were determined using a Reichert Kofler thermopan or in capillary tubes on a Büchi 510 apparatus and are uncorrected. IR spectra were recorded on a Perkin-Elmer 1640FT spectrophotometer. ${ }^{1} \mathrm{H}$ and ${ }^{13} \mathrm{C}$ NMR spectra were recorded on a Bruker AMX spectrometer at 300 and 75.47 MHz, respectively, using TMS as internal standard (chemical shifts in $\delta$ values, $J$ in $\mathrm{Hz}$ ). Mass spectra were obtained using a Hewlett Packard 5988A spectrometer. Elemental analyses were performed using a Perkin-Elmer 240B microanalyser and were within $\pm 0.4 \%$ of calculated values in all cases. Silica gel (Merck 60, 230-00 mesh) was used for flash chromatography (FC). Analytical thin 
layer chromatography (TLC) was performed on plates precoated with silica gel (Merck 60 F254, 0.25 $\mathrm{mm})$.

General procedure for the preparation of 3,4-cycloalkane-7-hydroxycoumarins $(1,2,11)$. 2Methylresorcinol (80.554 mmol) was dissolved in $12 \mathrm{M} \mathrm{H}_{2} \mathrm{SO}_{4}(120 \mathrm{~mL})$. The appropriate ethyl 2oxocycloalkanecarboxylate $(96.65 \mathrm{mmol})$ was added and the solution was stirred at room temperature for $15 \mathrm{~h}$. The reaction mixture was then poured into ice/water $(100 \mathrm{~mL})$ and the precipitate was recovered by filtration and washed with water to yield the desired compound, which was purified by crystallization.

8-Methyl-3,4-cyclopentene-7-hydroxycoumarin (1). Yield 82\%; mp $259{ }^{\circ} \mathrm{C}$ (EtOH); ${ }^{1} \mathrm{H}$ NMR (DMSO-d $\left.{ }_{6}\right) 2.07\left(\mathrm{~m}, 2 \mathrm{H}, \mathrm{CH}_{2} \underline{\mathrm{CH}}_{2} \mathrm{CH}_{2}\right), 2.16(\mathrm{~s}, 3 \mathrm{H}, \mathrm{Me}-\mathrm{C} 8), 2.70(\mathrm{t}, J=7.3,2 \mathrm{H}), 2.98(\mathrm{t}, J=7.5,2 \mathrm{H})$, $6.84(\mathrm{~d}, J=8.4,1 \mathrm{H}, \mathrm{H}-6), 7.24(\mathrm{~d}, J=8.4,1 \mathrm{H}, \mathrm{H}-5), 10.30(\mathrm{~s}, 1 \mathrm{H}, \mathrm{OH}) ;{ }^{13} \mathrm{C}$ NMR (DMSO- $\left.\mathrm{d}_{6}\right) 8.21$ (Me-C8), $22.03\left(\mathrm{CH}_{2} \mathrm{CH}_{2} \mathrm{CH}_{2}\right), 29.88,31.60,110.57$ (C8), 110.63, 111.66 (C6), 121.89, 123.00 (C5), 153.35, 157.02, 158.25, 159.46 (C2); IR 3362, 2920, 1682, 1577, 1275, 1081, 812; MS m/z 217 ([M + 1] $\left.]^{+}, 14\right), 216\left(\mathrm{M}^{+}, 100\right), 188(58), 187$ (65), 145 (6), 128 (3). Anal. $\left(\mathrm{C}_{13} \mathrm{H}_{12} \mathrm{O}_{3}\right) \mathrm{C}, \mathrm{H}$.

8-Methyl-3,4-cyclohexene-7-hydroxycoumarin (2). Yield 71\%; mp 279-280 ${ }^{\circ} \mathrm{C}(\mathrm{EtOH}) ;{ }^{1} \mathrm{H}$ NMR (DMSO-d $)_{6} 1.69\left(\mathrm{~m}, 4 \mathrm{H}, \mathrm{CH}_{2}\left(\mathrm{CH}_{2}\right)_{2} \mathrm{CH}_{2}\right), 2.11(\mathrm{~s}, 3 \mathrm{H}, \mathrm{Me}-\mathrm{C} 8), 2.35(\mathrm{~m}, 2 \mathrm{H}), 2.64(\mathrm{~m}, 2 \mathrm{H}), 6.79(\mathrm{~d}, J=$

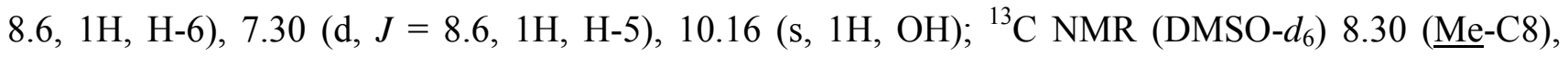
21.29, 21.61, $23.78\left(\underline{\mathrm{CH}}_{2}-\mathrm{C} 4\right), 24.95,110.61$ (C8), 111.87 (C6), 112.25, 118.27, 121.78 (C5), 148.13, 151.40, 157.94, 161.45 (C2); IR 3275, 2932, 1676, 1606, 1374, 1266, 1102, 804; MS m/z $231\left([\mathrm{M}+1]^{+}\right.$, 15), $230\left(\mathrm{M}^{+}, 100\right), 215$ (47), 202 (33), 174 (60). Anal. $\left(\mathrm{C}_{14} \mathrm{H}_{14} \mathrm{O}_{3}\right) \mathrm{C}, \mathrm{H}$.

5-Hydroxy-4-methyl-7-methoxycoumarin (11). Yield 50\%; mp $260{ }^{\circ} \mathrm{C}(\mathrm{EtOH}) ;{ }^{1} \mathrm{H}$ NMR (DMSOd $) 2.50(\mathrm{~d}, J=1.1,3 \mathrm{H}, \mathrm{Me}), 3.76$ (s, 3H, MeO), 5.93 (d, $J=1.1,1 \mathrm{H}, \mathrm{H}-3), 6.31$ (d, $J=2.4,1 \mathrm{H}, \mathrm{H}-6)$, $6.42(\mathrm{~d}, J=2.4,1 \mathrm{H}, \mathrm{H}-8), 10.70(\mathrm{~s}, 1 \mathrm{H}, \mathrm{OH}) ;{ }^{13} \mathrm{C}$ NMR (DMSO-d $) 23.71$ (Me-C4), 55.90 ( $\underline{\mathrm{MeO}-\mathrm{C} 8)}$, 93.11 (C8), 98.40 (C6), 103.52 (C4a), 110.22 (C3), 155.21 (C4), 156.83 (C8a), 158.11 (C5), 160.31 
(C2), 162.62 (C7); IR 3168, 2940, 1684, 1629, 1598, 1396, 1288, 1209, 1074, 846; MS m/z 207 ([M + $\left.1]^{+}, 13\right), 206\left(\mathrm{M}^{+}, 100\right), 178(86), 163(69), 135$ (10). Anal. $\left(\mathrm{C}_{11} \mathrm{H}_{10} \mathrm{O}_{4}\right) \mathrm{C}, \mathrm{H}$.

General procedure for the preparation of oxoether derivatives 3, 4, 5, 6, 7 and 12. To a solution of the substituted 7-hydroxycoumarin derivatives $\mathbf{1}, \mathbf{2}$ or $\mathbf{1 1}(62 \mathrm{mmol})$ in dry acetone $(300 \mathrm{~mL})$ were added $\mathrm{K}_{2} \mathrm{CO}_{3}(4 \mathrm{~g})$ and the corresponding 2-chloroketones or 2,3-dibromopropene (120 mmol). The mixture was heated under reflux for $24 \mathrm{~h}$. The mixture was cooled and the solid residue was filtered off. The solvent was removed under reduced pressure and the crude product was purified by FC and/or crystallization to give the desired compound.

7-Acetonyloxy-3,4-cyclopentene-8-methylcoumarin (3). 76\%; mp $144{ }^{\circ} \mathrm{C} ;{ }^{1} \mathrm{H} \mathrm{NMR}\left(\mathrm{CDCl}_{3}\right) 2.18$ (m, $2 \mathrm{H}, \mathrm{CH}_{2} \mathrm{CH}_{2} \mathrm{CH}_{2}$ ), 2.34 (s, 3H, MeCO), 2.38 (s, 3H, Me-C8), 2.87 (t, $J=7.5,2 \mathrm{H}$ ), 3.01 (t, $J=5.9$, $2 \mathrm{H}), 4.63\left(\mathrm{~s}, 2 \mathrm{H}, \mathrm{CH}_{2} \mathrm{O}\right), 6.64(\mathrm{~d}, J=8.6,1 \mathrm{H}, \mathrm{H}-6), 7.21(\mathrm{~d}, J=8.6,1 \mathrm{H}, \mathrm{H}-5) ;{ }^{13} \mathrm{C} \mathrm{NMR}\left(\mathrm{CDCl}_{3}\right) 9.01$ (Me-C8), $22.86\left(\mathrm{CH}_{2} \mathrm{CH}_{2} \mathrm{CH}_{2}\right), 27.10$ ( $\left.\underline{\mathrm{MeCO}}\right), 30.76,32.40,73.74\left(\mathrm{CH}_{2} \mathrm{O}\right), 107.73$ (C6), 113.69, 114.87, 122.98 (C5), 125.29, 153.72, 156.73, 157.98, 160.78 (C2), 205.29 (MeCO); IR 2956, 1733, 1706, 1615, 1376, 1286, 1120, 805; MS m/z 273 ([M + 1] $\left.]^{+}, 18\right), 272\left(\mathrm{M}^{+}, 100\right), 229(46), 215(43), 187$ (33), 128 (44). Anal. $\left(\mathrm{C}_{16} \mathrm{H}_{16} \mathrm{O}_{4}\right) \mathrm{C}, \mathrm{H}$.

7-Acetonyloxy-3,4-cyclohexene-8-methylcoumarin (4). Yield 79\%; mp 161-162 ${ }^{\circ} \mathrm{C} ;{ }^{1} \mathrm{H}$ NMR $\left(\mathrm{CDCl}_{3}\right) 1.81$ (m, 4H, $\left.\mathrm{CH}_{2}\left(\mathrm{CH}_{2}\right)_{2} \mathrm{CH}_{2}\right), 2.33$ (s, 3H, MeCO), 2.37 (s, 3H, Me-C8), 2.54 (m, 2H), 2.72 $(\mathrm{m}, 2 \mathrm{H}), 4.62\left(\mathrm{~s}, 2 \mathrm{H}, \mathrm{CH}_{2} \mathrm{O}\right), 6.64(\mathrm{~d}, J=8.8,1 \mathrm{H}, \mathrm{H}-6), 7.34(\mathrm{~d}, J=8.8,1 \mathrm{H}, \mathrm{H}-5) ;{ }^{13} \mathrm{C} \mathrm{NMR}\left(\mathrm{CDCl}_{3}\right)$ 8.39 ( $\underline{\mathrm{Me}}-\mathrm{C} 8), 21.43,21.67,23.89,25.21,26.70$ ( $\underline{\mathrm{MeCO}}), 73.40\left(\mathrm{CH}_{2} \mathrm{O}\right), 107.18$ (C6), 114.24 (C8), 114.79, 120.97, 121.08 (C5), 147.13, 151.22, 157.09, 162.05 (C2), 205.13 (MeCOO); IR 2942, 1738, 1698, 1605, 1416, 1292, 1025, 803; MS m/z 287 ([M + 1] $\left.]^{+}, 38\right), 286\left(\mathrm{M}^{+}, 100\right), 271(12), 258(5), 243$ (60), 230 (21). Anal. $\left(\mathrm{C}_{17} \mathrm{H}_{18} \mathrm{O}_{4}\right) \mathrm{C}, \mathrm{H}$.

7-Acetonyloxy-3,4-cyclohexene-8-methoxycoumarin (5). Yield 71\%; mp 141-143 ${ }^{\circ} \mathrm{C} ;{ }^{1} \mathrm{H}$ NMR $\left(\mathrm{CDCl}_{3}\right) 1.82\left(\mathrm{~m}, 4 \mathrm{H}, \mathrm{CH}_{2}\left(\mathrm{C}_{2}\right)_{2} \mathrm{CH}_{2}\right), 2.31(\mathrm{~s}, 3 \mathrm{H}, \mathrm{MeCO}), 2.55(\mathrm{~m}, 2 \mathrm{H}), 2.72(\mathrm{~m}, 2 \mathrm{H}), 4.01(\mathrm{~s}, 3 \mathrm{H}$, 
$\mathrm{MeO}), 4.70\left(\mathrm{~s}, 2 \mathrm{H}, \mathrm{CH}_{2} \mathrm{O}\right), 6.73(\mathrm{~d}, J=8.9,1 \mathrm{H}, \mathrm{H}-6), 7.22(\mathrm{~d}, J=8.9,1 \mathrm{H}, \mathrm{H}-5) ;{ }^{13} \mathrm{C} \mathrm{NMR}\left(\mathrm{CDCl}_{3}\right)$ 21.90, 22.18, 24.53, 25.86, $27.12(\underline{\mathrm{MeCO}}), 62.23(\mathrm{MeO}), 74.67\left(\mathrm{CH}_{2} \mathrm{O}\right), 110.50(\mathrm{C} 6), 116.66,118.65$ (C5), 122.22, 137.18, 147.03, 147.70, 152.83, 161.85 (C2), 205.22 (Meㅁ); IR 2941, 1734, 1697, 1609, 1302, 1115, 795; MS m/z 302 (M+100), 259 (26), 245 (55), 214 (17), 128 (14). Anal. $\left(\mathrm{C}_{17} \mathrm{H}_{18} \mathrm{O}_{5}\right) \mathrm{C}, \mathrm{H}$.

7-(ק-Bromoallyloxy)-3,4-cyclopentene-8-methylcoumarin (6). Yield 78\%; mp $140{ }^{\circ} \mathrm{C} ;{ }^{1} \mathrm{H}$ NMR $\left(\mathrm{CDCl}_{3}\right) 2.19$ (m, 2H, $\left.\mathrm{CH}_{2} \mathrm{C}_{2} \mathrm{CH}_{2}\right), 2.38$ (s, 3H, Me-C8), 2.90 (t, J= 7.5, 2H, $\left.\mathrm{CH}_{2}-\mathrm{C} 4\right), 3.03$ (t, J = 7.6, $\left.2 \mathrm{H}, \mathrm{CH}_{2}-\mathrm{C} 3\right), 4.72\left(\mathrm{~s}, 2 \mathrm{H}, \mathrm{CH}_{2} \mathrm{O}\right), 5.71(\mathrm{~d}, J=2.1,1 \mathrm{H}, \mathrm{C}=\mathrm{CH}), 6.01(\mathrm{~d}, J=2.1,1 \mathrm{H}, \mathrm{C}=\mathrm{CH}), 6.77(\mathrm{~d}, J$ $=8.6,1 \mathrm{H}, \mathrm{H}-6), 7.23(\mathrm{~d}, J=8.6,1 \mathrm{H}, \mathrm{H}-5) ;{ }^{13} \mathrm{C} \mathrm{NMR}\left(\mathrm{CDCl}_{3}\right) 8.61(\underline{\mathrm{Me}}-\mathrm{C} 8), 22.56\left(\mathrm{CH}_{2} \underline{\mathrm{CH}}_{2} \mathrm{CH}_{2}\right)$, $30.40\left(\underline{\mathrm{CH}}_{2}-\mathrm{C} 4\right), 32.07\left(\underline{\mathrm{CH}}_{2}-\mathrm{C} 3\right), 72.02\left(\mathrm{CH}_{2} \mathrm{O}\right), 108.09$ (C6), $113.26(\mathrm{C} 8), 114.81,118.00\left(\underline{\mathrm{CH}}_{2}=\mathrm{C}\right)$, 122.47 (C5), 124.94, 126.52, 153.40, 156.40, 157.63, 160.58 (C2); IR 2919, 1716, 1611, 1373, 1282, 1109, 803; MS m/z (\%): $336\left([\mathrm{M}+2]^{+}, 12\right), 335\left(\mathrm{M}^{+}, 12\right), 255$ (76), 215 (29), 187 (100), 128 (15). Anal. $\left(\mathrm{C}_{16} \mathrm{H}_{15} \mathrm{BrO}_{3}\right) \mathrm{C}, \mathrm{H}$.

7-(B-Bromoallyloxy)-3,4-cyclohexene-8-methylcoumarin (7). Yield $76 \%$; mp $135-136{ }^{\circ} \mathrm{C} ;{ }^{1} \mathrm{H}$ $\mathrm{NMR}\left(\mathrm{CDCl}_{3}\right) 1.82\left(\mathrm{~m}, 4 \mathrm{H}, \mathrm{CH}_{2}\left(\mathrm{CH}_{2}\right)_{2} \mathrm{CH}_{2}\right), 2.35$ (s, 3H, Me-C8), 2.56 (m, 2H, $\left.\mathrm{CH}_{2}-\mathrm{C} 4\right), 2.72$ (m, 2H, $\left.\mathrm{CH}_{2}-\mathrm{C} 3\right), 4.71\left(\mathrm{~s}, 2 \mathrm{H}, \mathrm{CH}_{2} \mathrm{O}\right), 5.76(\mathrm{~d}, J=1.6,1 \mathrm{H}, \mathrm{C}=\mathrm{CH}), 6.01(\mathrm{~d}, J=1.6,1 \mathrm{H}, \mathrm{C}=\mathrm{CH}), 6.75(\mathrm{~d}, J=$ 8.8, 1H, H-6), 7.35 (d, $J=8.8,1 \mathrm{H}, \mathrm{H}-5) ;{ }^{13} \mathrm{C}$ NMR $\left(\mathrm{CDCl}_{3}\right) 8.78(\underline{\mathrm{Me}}-\mathrm{C} 8), 21.86\left(\underline{\mathrm{CH}}_{2}-\mathrm{CH}_{2} \mathrm{C} 3\right), 22.11$ $\left(\underline{\mathrm{CH}}_{2}-\mathrm{CH}_{2} \mathrm{C} 4\right), 24.31\left(\underline{\mathrm{CH}}_{2}-\mathrm{C} 3\right), 25.65\left(\underline{\mathrm{CH}}_{2}-\mathrm{C} 4\right), 72.36\left(\underline{\mathrm{CH}}_{2} \mathrm{O}\right), 108.32$ (C6), 114.80 (C8), 115.04, $118.36\left(\underline{\mathrm{CH}}_{2}=\mathrm{C}\right), 121.26,121.35$ (C5), 127.03, 147.64, 151.57, 157.47, 162.60 (C2); IR 3071, 2935, 1708, 1605, 1114, 755; MS m/z 350 ([M + 2] $\left.]^{+}, 4\right), 349\left(\mathrm{M}^{+}, 22\right), 269$ (89), 229 (100), 201 (58), 187 (40). Anal. $\left(\mathrm{C}_{17} \mathrm{H}_{17} \mathrm{BrO}_{3}\right) \mathrm{C}, \mathrm{H}$.

5-Acetonyloxy-4-methyl-7-methoxycoumarin (12). Yield 95\%; mp 236-237 ${ }^{\circ} \mathrm{C} ;{ }^{1} \mathrm{H} \mathrm{NMR}\left(\mathrm{CDCl}_{3}\right)$ $2.28(\mathrm{~s}, 3 \mathrm{H}, \mathrm{MeCO}), 2.64(\mathrm{~d}, J=1.2,3 \mathrm{H}, \mathrm{Me}-\mathrm{C} 4), 3.83(\mathrm{~s}, 3 \mathrm{H}, \mathrm{MeO}), 4.66\left(\mathrm{~s}, 2 \mathrm{H}, \mathrm{CH}_{2} \mathrm{O}\right), 6.01(\mathrm{~d}, J=$ $1.2,1 \mathrm{H}, \mathrm{H}-3), 6.11(\mathrm{~d}, J=2.3,1 \mathrm{H}, \mathrm{H}-8), 6.48(\mathrm{~d}, J=2.3,1 \mathrm{H}, \mathrm{H}-6) ;{ }^{13} \mathrm{C}$ NMR $\left(\mathrm{CDCl}_{3}\right) 24.58$ (的-C4), $26.70(\underline{\mathrm{MeCO}}), 55.82(\mathrm{MeO}) 73.29\left(\mathrm{CH}_{2} \mathrm{O}\right), 94.27$ (C8), 96.41 (C6), 105.08, 111.99 (C3), 154.05, 
156.93, 157.23, 160.85, 162.58 (C2), 202.45 (Meㅁ); IR 2945, 1733, 1625, 1592, 1385, 1336, 1118 , 847; MS m/z $263\left([\mathrm{M}+1]^{+}, 15\right), 262\left(\mathrm{M}^{+}, 100\right), 234$ (45), 219 (20), 205 (44), 191 (20). Anal. $\left(\mathrm{C}_{14} \mathrm{H}_{14} \mathrm{O}_{5}\right) \mathrm{C}, \mathrm{H}$.

General procedure for the preparation of 3,4-benzocoumarins 8-10. To a solution of the acyclic ether $4,5^{30}$ or $7(0.30 \mathrm{mmol})$ in toluene $(15 \mathrm{~mL})$ was added DDQ $(0.60 \mathrm{mmol})$. The solution was heated under reflux for $5 \mathrm{~h}$. The mixture was cooled, the precipitate filtered off and the solvent evaporated under reduced pressure. The resulting residue was purified by FC to give the desired compound.

3,4-Benzo-7-acetonyloxy-8-methylcoumarin (8). Yield 64\%; mp $172-174{ }^{\circ} \mathrm{C} ;{ }^{1} \mathrm{H}$ NMR $\left(\mathrm{CDCl}_{3}\right)$ 2.35 (s, 6H, Me-C8, MeCO), 4.60 (s, 2H, $\mathrm{CH}_{2} \mathrm{O}$ ), 6.63 (d, J = 8.8, 1H, H-6), 7.46 (m, 1H, C프-CHC3), 7.73 (m, 2H, H-5, Cㅂ-CHC4), 7.91 (d, $J=8.0,1 \mathrm{H}, \mathrm{CH}$ C4), 8.28 (d, J = 7.9, 1H, CH-C3); ${ }^{13} \mathrm{C}$ NMR $\left(\mathrm{CDCl}_{3}\right) 8.45$ ( $\left.\underline{\mathrm{Me}}-\mathrm{C} 8\right), 26.67(\underline{\mathrm{MeCO}}), 73.19\left(\mathrm{CH}_{2} \mathrm{O}\right), 107.43$ (C6), 111.95, 114.95, 119.72, 120.44

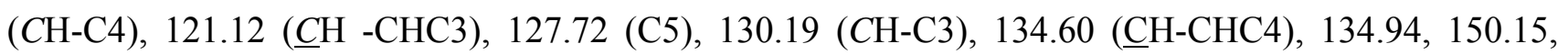
156.95, 161.13 (C2), 204.98 (MeCO); IR 2925, 1716, 1607, 1468, 1284, 1125, 766; MS m/z 283 ([M + $\left.1]^{+}, 18\right), 282\left(\mathrm{M}^{+}, 100\right), 239$ (60), 225 (43), 181 (51), 152 (47). Anal. $\left(\mathrm{C}_{17} \mathrm{H}_{14} \mathrm{O}_{4}\right) \mathrm{C}, \mathrm{H}$.

3,4-Benzo-7-acetonyloxy-8-methoxycoumarin (9). Yield 56\%; mp 172-174 ${ }^{\circ} \mathrm{C} ;{ }^{1} \mathrm{H}$ NMR $\left(\mathrm{CDCl}_{3}\right)$ $2.33(\mathrm{~s}, 3 \mathrm{H}, \mathrm{MeCO}), 4.05(\mathrm{~s}, 3 \mathrm{H}, \mathrm{MeO}), 4.72\left(\mathrm{~s}, 2 \mathrm{H}, \mathrm{CH}_{2} \mathrm{O}\right), 6.80(\mathrm{~d}, J=9.0,1 \mathrm{H}, \mathrm{H}-6), 7.53$ (t, J = 8.1,

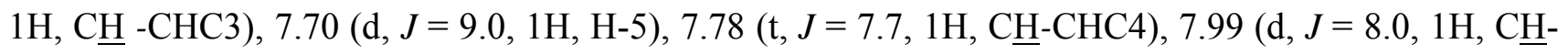
C4), $8.36(\mathrm{~d}, J=7.9,1 \mathrm{H}, \mathrm{C} \underline{\mathrm{H}}-\mathrm{C} 3) ;{ }^{13} \mathrm{C}$ NMR $\left(\mathrm{CDCl}_{3}\right) 27.01(\underline{\mathrm{MeCO}}), 62.15(\mathrm{MeO}), 74.62\left(\mathrm{CH}_{2} \mathrm{O}\right)$,

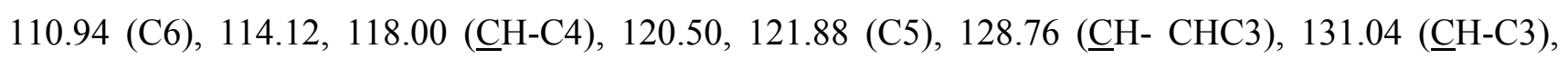
135.29, 135.36 (ㅁH-CHC4), 137.94, 146.26, 152.78, 161.07 (C2), 205.12 (Meㅁ); IR 2935, 1730, 1608, 1475, 1304, 1121, 812; MS m/z 299 ([M + 1] $\left.]^{+}, 18\right), 298\left(\mathrm{M}^{+}, 100\right), 255$ (27), 241 (67), 197 (25), 170 (19). Anal. $\left(\mathrm{C}_{17} \mathrm{H}_{14} \mathrm{O}_{5}\right) \mathrm{C}, \mathrm{H}$.

3,4-Benzo-7-(b-bromoallyloxy)-8-methylcoumarin (10). Yield 82\%; mp $128{ }^{\circ} \mathrm{C} ;{ }^{1} \mathrm{H} \mathrm{NMR}\left(\mathrm{CDCl}_{3}\right)$ $2.38(\mathrm{~s}, 3 \mathrm{H}, \mathrm{Me}-\mathrm{C} 8), 4.72\left(\mathrm{~s}, 2 \mathrm{H}, \mathrm{CH}_{2} \mathrm{O}\right), 5.72(\mathrm{~d}, J=1.9,1 \mathrm{H}, \mathrm{C}=\mathrm{CH}), 6.03(\mathrm{~d}, J=1.9,1 \mathrm{H}, \mathrm{C}=\mathrm{CH})$, 
$6.80(\mathrm{~d}, J=8.8,1 \mathrm{H}, \mathrm{H}-6), 7.49(\mathrm{~m}, 1 \mathrm{H}, \mathrm{C} \underline{\mathrm{H}}-\mathrm{CHC} 4), 7.78(\mathrm{~m}, 1 \mathrm{H}+1 \mathrm{H}, \mathrm{C} \underline{\mathrm{H}}-\mathrm{CHC} 3+\mathrm{H}-5), 7.98(\mathrm{~d}, J=$ 8.0, 1H, CH-C3), 8.34 (d, J=8.0, 1H, CH-C4); ${ }^{13} \mathrm{C}$ NMR $\left(\mathrm{CDCl}_{3}\right) 8.95(\underline{\mathrm{Me}}-\mathrm{C} 8), 72.33\left(\mathrm{CH}_{2} \mathrm{O}\right), 108.68$

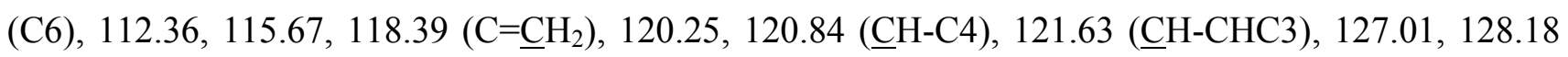
(C5), 130.75 (ㅁH-CHC4), 135.12 (ㅁH-C3), 135.57, 150.66, 157.49, 161.78 (C2); IR 2921, 1726, 1608, 1470, 1283, 1115, 891, 766; MS m/z 347 ([M + 2] $\left.]^{+}, 2\right), 345\left(\mathrm{M}^{+}, 12\right), 344$ (13), 265 (60), $225(100), 171$ (17). Anal. $\left(\mathrm{C}_{17} \mathrm{H}_{13} \mathrm{BrO}_{3}\right) \mathrm{C}, \mathrm{H}$.

Preparation of 7-Methoxy-4-methyl-4'-methylfuro[5,6-g]coumarin 13. To a solution of the corresponding ketoether $12(3.39 \mathrm{mmol})$ in ethyl alcohol $(200 \mathrm{~mL})$ was added 0.1M NaOH $(200 \mathrm{~mL})$. The mixture was heated under reflux for $12 \mathrm{~h}$, acidified with $\mathrm{HCl}$ and concentrated to half volume and left overnight. The resulting precipitate was filtered off and purified by FC to give the desired compound (13). Yield 52\%; mp $212{ }^{\circ} \mathrm{C} ;{ }^{1} \mathrm{H}$ NMR $\left(\mathrm{CDCl}_{3}\right) 2.35$ (d, $\left.J=1.3,3 \mathrm{H}, \mathrm{Me}-\mathrm{C} 4\right), 2.68(\mathrm{~d}, J=$ 1.2, 3H, Me-C4), 3.95 (s, 3H, MeO), 6.09 (d, J = 1.2, 1H, H-3), 6.61 (s, 1H, H-8), 7.35 (d, J = 1.3, 1H, $\left.\mathrm{H}-5{ }^{\prime}\right) ;{ }^{13} \mathrm{C} \mathrm{NMR}\left(\mathrm{CDCl}_{3}\right) 9.71$ ( $\underline{\mathrm{Me}-\mathrm{C} 4}$ ), 21.93 (Me-C4), 55.92 (MeO), 93.64 (C8), 102.00, 111.10 (C3), 115.34, 116.22, 140.61 (C5'), 152.26, 153.72, 153.89, 157.42, 161.45 (C2); IR 2989, 1717, 1619, 1606, 1358, 1166, 1124, 815; MS m/z $245\left([\mathrm{M}+1]^{+}, 15\right), 244\left(\mathrm{M}^{+}, 100\right), 216(41), 201(87)$. Anal. $\left(\mathrm{C}_{14} \mathrm{H}_{11} \mathrm{O}_{4}\right)$ C, H.

Biological assay. Enzymatic MAO-A and MAO-B activity of compounds was determined by a fluorimetric method following a previously described protocol. ${ }^{24}$ Briefly, $0.1 \mathrm{~mL}$ of sodium phosphate buffer (0.05 M, pH 7.4) containing various concentrations of the test drugs and appropriate amounts of recombinant hMAO-A or hMAO-B and adjusted to obtain in our experimental conditions the same reaction velocity in the presence of both isoforms were incubated for $15 \mathrm{~min}$ at $37^{\circ} \mathrm{C}$ in a flat-blackbottom 96-well microtest plate placed in the dark fluorimeter chamber. After this incubation period, the reaction was started by adding (final concentrations) $200 \mu \mathrm{M}$ of 10-acetyl-3,7-dihydroxyphenoxazine reagent (Amplex Red assay kit), $1 \mathrm{U} / \mathrm{ml}$ horseradish peroxidase and $1 \mathrm{mM}$ p-tyramine. The production 
of $\mathrm{H}_{2} \mathrm{O}_{2}$ and, consequently, of resorufin was quantified at $37{ }^{\circ} \mathrm{C}$ in a Multi-Detection microplate fluorescence reader (FLX800) based on the fluorescence generated (excitation, $545 \mathrm{~nm}$, emission, 590 $\mathrm{nm}$ ) over a $15 \mathrm{~min}$ period, during which the fluorescence increased linearly.

Control experiments were carried out simultaneously by replacing the test drugs (new compounds and reference inhibitors) with appropriate dilutions of the vehicles. In addition, the possible capacity of the above test drugs to modify the fluorescence generated in the reaction mixture due to non-enzymatic inhibition was determined by adding these drugs to solutions containing only the Amplex Red reagent in a sodium phosphate buffer.

To determine the kinetic parameters of hMAO-A and hMAO-B $\left(\mathrm{K}_{\mathrm{m}}\right.$ and $\left.\mathrm{V}_{\max }\right)$, the corresponding enzymatic activity of both isoforms was evaluated (under the experimental conditions described above) in the presence of a number of $p$-tyramine concentrations.

The specific fluorescence emission (used to obtain the final results) was calculated after subtraction of the background activity, which was determined from vials containing all components except the MAO isoforms, which were replaced by a sodium phosphate buffer solution.

Acknowledgment. We are grateful to the Xunta de Galicia (INCITE07PXI203030ES, PGIDIT05BTF20302PR and INCITE08PXIB203022PR) and Ministerio de Sanidad y Consumo (FIS PI061537 and PI061457) for financial support.

\section{References}

(1) Dostert, P.; Strolin Benedetti, M.; Jafre, M. Structural modifications in oxazolidinone series leading to type A or B selective monoamine oxidase inhibitors. In Monoamine Oxidase: Basic and Clinical Frontiers; Kamijo, K.; Usdin, E.; Nagausu, T., Eds.; Excerpta Medica: Amsterdam, 1982; pp 197-208.

(2) Singer, T. P. Monoamine oxidases. In Chemistry and Biochemistry of Flavoenzymes (II). Muller, F., Ed.; CRC Press: London, 1991; pp 437-470.

(3) De Colibus, L.; Li, M., Binda, C.; Lustig, A.; Edmondson, D.E.; Mattevi, A. Three-dimensional structure of human monoamine oxidase A (MAO A): Relation to the structures of rat MAO A and human MAO B. Proc. Natl. Acad. Sci. U.S.A. 2005, 102, 
12684-12689.

(4) Binda, C.; Li, M.; Hubálek, F.; Restelli, N.; Edmondson, D. E.; Mattevi, A. Insights into the mode of inhibition of human mitochondrial monoamine oxidase B from high-resolution crystal structures. Proc. Natl. Acad. Sci. U. S. A. 2003, 100, $9750-9755$.

(5) Yamada,M.; Yasuhara, H. Clinical pharmacology of MAO inhibitors: safety and future. Neurotoxicology 2004, $25,11-20$.

(6) Rudorfer, M. V.; Potter, V. Z.; Antidepressants. A comparative review of the clinical pharmacology and therapeutic use of the "newer" versus the "older" drugs. Drugs 1989, 37, 713-738.

(7) Palhagen, S.; Heinonen, E.; Hagglund, J.; Kaugesaar, T.; Maki-Ikola, O.; Palm, R. Selegiline slows the progression of the symptoms of Parkinson disease. Neurology 2006, 66, 1200-1206.

(8) Guay, D. R. Rasagiline (TVP-1012): a new selective monoamine oxidase inhibitor for Parkinson's disease. Am. J. Geriatr. Pharmacother. 2006, 4, 330-346.

(9) Riederer, P. Danielczyk, W.; Grunblatt, E. Monoamine oxidase-B inhibition in Alzheimer's desease. Neurotoxicology 2004, 25, 271-277.

(10) Youdim, M. B. H.; Fridkin, M.; Zheng, H. Novel bifunctional drugs targeting monoamine oxidase inhibition and iron chelation as an approach to neuroprotection in Parkinson's disease and other neurodegenerative diseases. J. Neural Transm. 2004, 111, 14551471.

(11) Cesura, A. M.; Pletscher, A. The new generation of monoamine oxidase inhibitors. Prog. Drug Res. 1992, 38, 171-297.

(12) Youdim, M. B. The advent of selective monoamine oxidase A inhibitor antidepressants devoid of the cheese reaction. Acta Psychiatr. Scand. Suppl. 1995, 386, 5-7.

(13) Edmondson, D. E.; Mattevi, A.; Binda, C.; Li, M.; Hubalek, F. Structure and mechanism of monoamine oxidase. Curr. Med. Chem. 2004, 11, 1983-1993.

(14) Tipton, K. F.; Boyce, S.; O'Sullivan, J.; Davey, G. P.; Healy, J. Monoamine oxidases: Certainties and uncertainties. Curr. Med. Chem. 2004, 11, 1965-1982.

(15) Edmondson, D. E.; Binda, C.; Mattevi, A. Structural insights into the mechanism of amine oxidation by monoamine oxidases A and B. Arch. Biochem. Biophys. 2007, 464, 269-276.

(16) Binda, C.; Hubalek, F.; Li, M.; Herzig, Y.; Sterling, J.; Edmondson, D. E.; Mattevi, A. Crystal Structures of Monoamine Oxidase B in Complex with Four Inhibitors of the N-Propargylaminoindane Class. J. Med. Chem. 2004, 47, 1767-1774.

(17) Chimenti, F.; Maccioni, E.; Secci, D.; Bolasco, A.; Chimenti, P.; Granese, A.; Befani, O.; Turini, P.; Alcaro, S.; Ortuso, F; Cirilli, R.; La Torre, F.; Cardia, M. C.; Distinto, S. Synthesis, Molecular Modeling Studies, and Selective Inhibitory Activity against Monoamine Oxidase of 1-Thiocarbamoyl-3,5-diaryl-4,5-dihydro-(1H)- pyrazole Derivatives. J. Med. Chem. 2005, 48, 7113-7122.

(18) Harkcom, W. T.; Bevan, D. R. Molecular docking of inhibitors into monoamine oxidase B. Biochem. Biophys. Res. Comm. 2007, 360, 401-406.

(19) Gallardo-Godoy, A.; Fierro, A.; McLean, T. H.; Castillo, M.; Cassels, B. K.; Reyes-Parada, M.; Nichols, D. E. Sulfur-Substituted $\alpha$-Alkyl Phenethylamines as Selective and Reversible MAO-A Inhibitors: Biological Activities, CoMFA Analysis, and Active Site Modeling. J. Med. Chem. 2005, 48, 2407-2419.

(20) Mevedev, A. E.; Veselovsky, A. V.; Shvedov, V. I.; Tikhonova, T. A.; Moskvitinia, T. A.; Fedotova, O. A.; Axenova, L. N.; Kamyshanskaya, A. Z.; Kirkel, A. Z. and Ivanov, A. Inhibition of Monoamino Oxidase by Pirlindole Analogues: 3D-QSAR and CoMFA Analysis. J. Chem. Inf. Comput. Sci. 1998, 38, 1137-1144.

(21) González-Díaz, H.; Molina-Ruiz, R.; Hernandez, I. MARCH-INSIDE.Version 3.0 (MARkov CHains INvariants for SImulation \& DEsign), 2007; Windows supported version released under request to the main author contact email: humberto.gonzalez@usc.es.

(22) González-Díaz, H.; Torres-Gómez, L. A.; Guevara, Y.; Almeida, M. S.; Molina, R.; Castañedo, N.; Santana, L.; Uriarte, E. Markovian chemicals "in silico" design (MARCH-INSIDE), a promising approach for computer-aided molecular design III: 2.5D indices for the discovery of antibacterials. J. Mol. Mod. 2005, 11, 116-123.

(23) Borges, F.; Roleira, F.; Mihazes, N.; Santana, L.; Uriarte, E. Simple coumarins and analogues in medicinal chemistry: occurrence, synthesis and biological activity. Curr. Med. Chem, 2005, 12, 887-916.

(24) Chimenti, F.; Secci, D.; Bolasco, A.; Chimenti, P.; Granese, A.; Befani, O.; Turini, P.; Alcaro, S.; Ortuso, F. Inhibition of 
monoamine oxidases by coumarin-3-acyl derivatives: biological activity and computational study. Bioorg. Med. Chem. Lett. 2004, 14, 3697-3703.

(25) Catto, M.; Nicolotti, O.; Leonetti, F.; Carotti, A.; Soto-Otero, R.; Mendez-Alvarez, E.; Carotti, A. Structural Insights into Monoamine Oxidase Inhibitory Potency and Selectivity of 7-Substituted Coumarins from Ligand- and Target-Based Approaches. J. Med. Chem. 2006, 49, 4912-4925.

(26) Binda, C.; Wang, J.; Pisani, L.; Caccia, C.; Carotti, A.; Salvati, P.; Edmondson, D. E.; Mattevi, A. Structures of Human Monoamine Oxidase B Complexes with Selective Noncovalent Inhibitors: Safinamide and Coumarin Analogs. J. Med. Chem. 2007, 50, 5848-5852.

(27) Carotti, A.; Altomare, C.; Catto, M.; Gnerre, C.; Summo, L.; De Marco, A.; Rose, S.; Jenner, P.; Testa, B. Lipophilicity Plays a Major Role in Modulating the Inhibition of Monoamine Oxidase B by 7-Substituted Coumarins. Chem. Biodiversity 2006, 3, 134149.

(28) Santana, L.; Uriarte, E.; González-Díaz, H.; Zagotto, G.; Soto-Otero, R.; Méndez-Alvarez, E. A QSAR Model for in silico Screening of MAO-A Inhibitors. Prediction, Synthesis, and Biological Assay of Novel Coumarins. J. Med. Chem. 2006, 49, 11491156.

(29) Yáñez, M.; Fraiz, N.; Cano, E.; Orallo, F. Inhibitory effects of cis- and trans-resveratrol on noradrenaline and 5-hydroxytryptamine uptake and on monoamine oxidase activity. Biochem. Biophys. Res. Comm. 2006, 344, 688-695.

(30) Dalla Via, L.; Uriarte, E.; Quezada, E.; Dolmella, A.; M. G. Ferlin, M. G.; Gia, O. Novel Pyrone Side Tetracyclic Psoralen Derivatives: Synthesis and Photobiological Activity. J. Med. Chem. 2003, 46, 3800-3810. 